\title{
Studying the Properties of Metric Anisotropy for Implementation the Directed Couplers in Optical Fiber
}

\author{
Al-Gawagzeh Mohammed Yousef \\ Department of Electrical Power Engineering, Faculty of Engineering, Balqa Applied University, \\ 19117 Salt, Jordan, gogazeh@bau.edu.jo
}

\begin{abstract}
This research will study the ability of directional coupler realization on the ground of double refraction in anisotropic spiral stowed fiber at room temperature, the importance of using the features of anisotropic media to minimize the attenuation and dispersion in the transmitted signals in the fiber optic system of transfer which caused by the power exchange between waves and the possibility to get calculation results for specific cases. And try to determine the half-length of the beat region ${ }_{1}$ for a certain composition of the spiral single mode optical fibers depending on the parameters of the spiral to construct spiral single mode optical fibers based on photo elasticity. Also in this research we will try to define specific parameters which can be taken as a basis for directional couplers design to uniformly separate the power of the optical signal and to obtain the condition of uniform power distribution between the main waves $\left(\mathrm{HE}_{11}{ }^{\mathrm{e}}\right.$ and $\left.\mathrm{HE}_{11}{ }^{\circ}\right)$.
\end{abstract}

$\underline{\text { Key words: Optical fiber, directional coupler, spiral, photo elasticity, power separation, attenuation }}$

\section{INTRODUCTION}

The using of anisotropic components for constructing the elements of fiber-optic transmission systems represents a great scientific interest. But there is serious problems of uniformity in the s signal power separation and minimization the losses in directional couplers, using the features of anisotropic media will improve these characteristics. One of the methods to create an anisotropic optical fiber is photo elasticity which caused by the deposition of the fiber in a spiral line of constant step and radius of twisting at room temperature. The double refraction in an anisotropic medium, owing to ordinary and extraordinary waves which deviate at different angles with respect to the axis of the fiber can be used for the signal power separation. To implement the directional couplers where the principle of operation is based on spatial dilution of power equality of basic waves in an anisotropic medium due to photo elasticity, it is necessary to have the full information such: behavior character of ordinary $\left(\mathrm{HE}_{11}{ }^{\circ}\right)$ and extraordinary $\left(\mathrm{HE}_{11}{ }^{\circ}\right)$ waves in an anisotropic medium, the value of the dielectric permittivity tensor elements, the features of power transfer between the main $\left(\mathrm{HE}_{11}{ }^{\mathrm{e}}\right.$ and $\left.\mathrm{HE}_{11}{ }^{\circ}\right)$ waves and the effect of photo elasticity on the polarization dispersion.

The listed features of Spiral Single Mode Optical Fibers (SSMOF) have not been thoroughly investigated and to analysis the possibility of introducing into optical fiber system of transfer where principle of operation is based on anisotropy using for uniform power separation, it is necessary to carry out a research that aim to determine a specific patterns of changes in the above parameters at different degrees of the anisotropy influence.

Literature review: There are a number of scientific researchs devoted to this issue. By AL-Gawagzeh (2016) Makarov (2004), the transient attenuation was studied in the exchange of powers between ordinary and extraordinary waves and the range of the parameter $\mathrm{A}=\bullet / 4 \bullet \mathrm{R}(\bullet$-spiral step, $\mathrm{R}$-spiral radius $)$ at which the necessary value of these losses was determined. By Makarov (2005) the possibilities of using the angular dispersion property in the anisotropic medium are analyzed to achieve the separation of signal power with help of birefringence (double refraction).

By Makarov (2004) and Arnold (2001), the parameters of an anisotropic self-focusing optical fiber were calculated. By Zhuck and Shulga (2004) and AL-Gawagzeh et al. (2009) the polarization dispersion of optical fiber that subjected to the influence of mechanical stresses have been carried out and expressions have been obtained for its calculation. By AL-Gawagzeh et al. (2009) AL-Gawagzeh and Rasoul (2014) Yousef (2016) AL-Gawagzeh (2016) Iorgach (2011) and Peter (2008), measurements of the polarized modal dispersion in the wavelength range (1510-1645 nm) due to the bending of the optical fiber have been made and the dependence of the dispersion value on the bending radius of the fiber has been determined. However, in the above mentioned 
research, it was not defined the conditions were the coupling powers between the main waves in the photo elastic of the fiber are equal to each other. It was not clearly defined in the devices design, in particular the directional coupler.

The purpose of this study is to determine the specific parameters that can be taken as a basis for designing the directional couplers for uniform power separation of optical signal.

\section{MATERIALS AND METHODS}

According to the Mauls law, the intensity of ordinary $I_{0}$ and extraordinary $I_{e}$ waves is related to the angles $\left(\bullet_{0}, \bullet_{e}\right)$ between the optical axis and the tension vectors $\overrightarrow{\mathrm{E}}_{\mathbf{e}}, \overline{\mathrm{E}}_{\mathbf{o}}$ by the following relationships (Makarov, 2005):

$$
I_{0}=I \cdot \sin ^{2} \cdot \beta_{0} ; I_{e}=I \cdot \cos ^{2} \cdot \beta_{e}
$$

where, $i$ is the intensity of the radiation before the bending of the fiber. Obviously with a uniform power distribution $\left(\mathrm{I}_{\mathrm{o}}=\mathrm{I}_{\mathrm{e}}\right)$, it is necessary to make $\left(\bullet_{0}=\bullet_{\mathrm{e}}=45^{\circ}\right)$ as shown on the next Fig. 1. Where, $\overrightarrow{\mathrm{E}}_{\mathrm{o}}, \overrightarrow{\mathrm{E}}_{\mathrm{e}}$ the intensity vectors of the ordinary and extraordinary waves. $\vec{x}(\xi)$ the optical axis.

The power ratio between the fundamental waves $\left(\mathrm{HE}_{11}{ }^{\mathrm{e}}\right.$ and $\left.\mathrm{HE}_{11}{ }^{\circ}\right)$ must be equal to unity. When a wave is injected into an anisotropic medium, the birefringence occurs and at the initial time moment, under the condition $\left(\bullet_{\mathrm{o}}=\bullet_{\mathrm{e}}=45^{\circ}\right)$, the power of extraordinary wave is equal to the power of ordinary wave. After passing through the (SSMOF), the power is exchanged between these waves and the direction of power transfer changes to the opposite after passing through the length of (SSMOF)

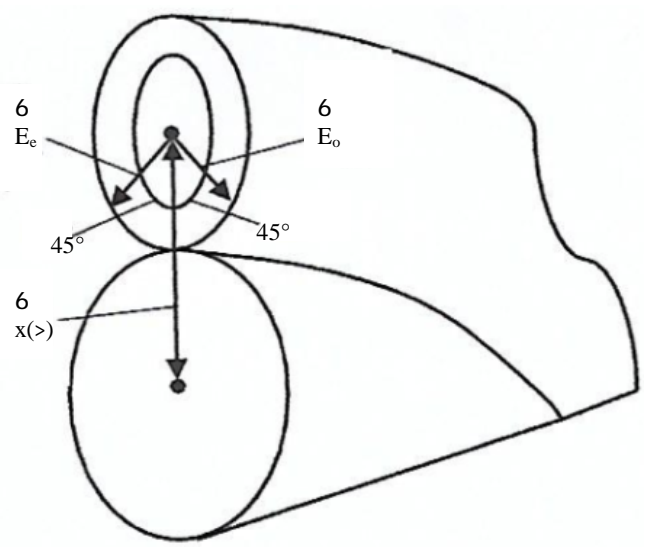

Fig. 1: The main wave's polarization when a signal entering into the (SSMOF) for equal power separation which equal to the half-length of the beat $\left(\bullet_{1}\right)$. Depending on the parameters of the spiral, the value of pumped power from extraordinary wave to the ordinary wave can be differing from the power that is pumped from the ordinary wave to the extraordinary wave. This task is needed to achieve an equality between transient attenuation $\left(\bullet_{\mathrm{nl}}{ }^{\mathrm{oe}}\left(\bullet_{1}\right)\right.$ and $\bullet_{\mathrm{nl}}{ }^{\mathrm{eo}}\left(\bullet_{1}\right)$ that mean, the optical power which transmitted by one of the waves during the passage of the half-length of the beat ${ }_{1}$ should be equal to the received optical power after passing the next section $\bullet_{1}$. In this case, after passing the length of $\left(2 \bullet_{1}\right)$ the power of extraordinary wave $\mathrm{HE}_{11}{ }^{\mathrm{e}}$ is equal to the power of the ordinary wave $\mathrm{HE}_{11}{ }^{\circ}$. Using the function of photo elasticity effect of power transfer from extraordinary wave to the ordinary:

$$
f(A)^{e 0}=\frac{A \sqrt{1+A^{2}}}{\left|A^{2}+A-1\right|} \cdot\{\}^{\mathrm{eo}}
$$

And the function of photo elasticity effect when the power transferring from an ordinary wave to an extraordinary:

$$
f(A)^{\mathrm{oe}}=\frac{\mathrm{A} \sqrt{1+\mathrm{A}^{2}}}{\left|\mathrm{~A}^{2}+\mathrm{A}-1\right|} \cdot\{\}^{\mathrm{oe}}
$$

where, \{\}$^{\text {eo }}$-the expression in formula (29) in Eq. 1 and \{\}$^{\circ e}$ the expression in the formula (35) by Makarov (2004). These functions show the dependence on the bending geometry of the optical fiber (fractional expressions) and the angles of polarization of the (SSMOF) material (expressions in curly brackets). Using the results that investigated and carried out by AL-Gawagzeh (2016) Makarov (2004), we obtain the condition of uniform power distribution between the main waves $\left(\mathrm{HE}_{11}{ }^{\mathrm{e}}\right.$ and $\left.\mathrm{HE}_{11}{ }^{\circ}\right)$ Eq. 4 :

$$
\alpha_{\mathrm{n} 1}^{\mathrm{eo}}\left(\xi_{1}\right)=\alpha_{\mathrm{n} 1}^{\mathrm{oe}}\left(\xi_{1}\right)
$$

If we apply expressions 2, 3 and also the calculated formulas for the transition attenuation that obtained by AL-Gawagzeh (2016) Makarov (2004), then the expression 4 can be reduces to the form:

$$
f(A)^{e o}=f(A)^{o e}
$$

Figure 2 show the dependences of the functions and on the dimensionless parameter. The functions curves of $\mathrm{f}(\mathrm{A})^{\mathrm{eo}}$ and $\mathrm{f}(\mathrm{A})^{\mathrm{oe}}$ gives a possibility to determine for which parameter values of (A) there will be a transient attenuation, so, we can see that for a positive value of 


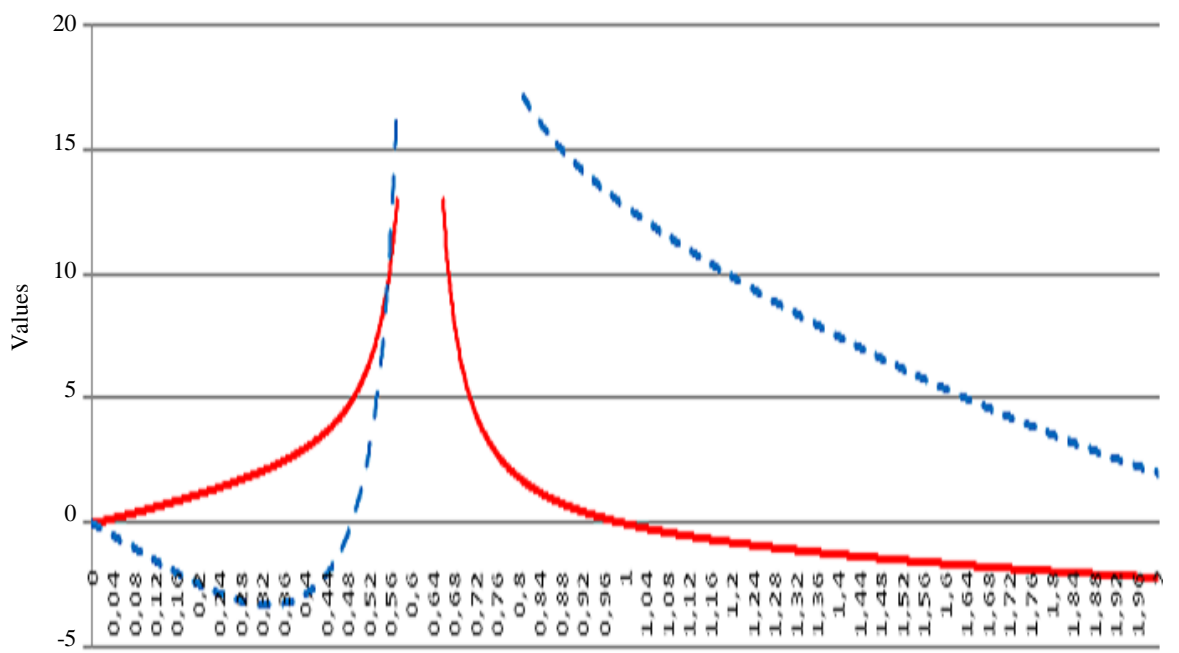

Fig. 2: Dependences of the photo elasticity functions $\mathrm{f}(\mathrm{A})^{\mathrm{ee}}$ (solid line) and $\mathrm{f}(\mathrm{A})^{\mathrm{oe}}$ (dashed line) on the parameter

$\mathrm{f}(\mathrm{A})^{\mathrm{e}}$, the power of the $\mathrm{HE}_{11}{ }^{\mathrm{e}}$ waves will be pumped to the wave $\mathrm{HE}_{11}{ }^{\circ}$ and for $\mathrm{f}(\mathrm{A})^{\mathrm{e}} \bullet 0$ there is practically no power transfer, similarly for $\mathrm{f}(\mathrm{A})^{\circ e}$,

As can be seen from the graph of Fig. 2, the condition of expression 5 is achieved for the values of A (from $0-2): A=0$ (bent along the arc of the circumference of optical fiber), $\mathrm{A}=0.553, \mathrm{~A}>2$. The half-length of the beat region is calculated by Makarov (2004):

$$
\xi=\frac{\sqrt{2} \lambda \cdot n_{1} \cdot R}{w} \cdot \frac{\left(1+4 A^{2}\right) \cdot \sqrt{1+A^{2}}}{\left|A^{2}+A-1\right|}
$$

For determining the half-length of the beat region for a certain composition of the (SSMOF) at the value of (A) parameter which corresponds to a uniform distribution of the signal power, it is necessary to determine the following parameters of the (SSMOF): spiral radius, step twisting, the number of turns, the length of the optical fiber, the length of the spiral where the optical fiber is deposited and also the angle of deviation of the extraordinary wave from the ordinary wave $(\bullet)$.

Since, the spatial spreading of the waves must be carried out, then the length of the optical fiber and the length of the spiral are:

$$
1=2 \cdot \xi_{1} \cdot \mathrm{m}, \mathrm{s}=\mathrm{k} \cdot \mathrm{\rho}
$$

Where:

$\mathrm{I}=$ The optical fiber length

$\mathrm{m}=$ An integer

$\mathrm{s}=$ The spiral length where the optical fiber is deposited

$\mathrm{k}=$ The number of spiral turns
Representing the length of optical fiber that spent on one turn in the form of a hypotenuse a rectangular triangle where one of the legs is equal to the step of twisting and the other length is the circle of the cylinder cross section where the winding is performed, then the total length of the optical fiber is:

$$
1=k \sqrt{\rho^{2}+(2 \pi R)^{2}}
$$

\section{RESULTS AND DISCUSSION}

Using the expression 7, the turn's number of (SSMOF) can be defined as:

$$
\mathrm{k}=\frac{2 \xi_{1} \cdot \mathrm{m}}{\sqrt{\rho^{2}+(2 \pi R)^{2}}}
$$

Figure 3 shows the construction of (SSMOF) that necessary for uniform signal power distribution. The next Table 1 show the parameters calculations results with a uniform power separation for some compositions of optical fiber with a core radius of $4.5 \mu \mathrm{m}, \mathrm{m}=1, \mathrm{~A}=0.553$ and a wavelength of $1.565 \mu \mathrm{m}$.

From simplicity point of view in realizing the uniform power separation based on an anisotropic (SSMOF), the case $\mathrm{A}=0$ (bent along the arc of the circle of fiber waveguide) and $\mathrm{A}>2$ is optimal, since, ordinary and extraordinary waves propagate autonomously without exchange of powers (AL-Gawagzeh, 2016; Makarov, 2004) and therefore, there are no strict requirements for determining the length of the (SSMOF) section where it is necessary to dilute both beams. If the condition $\cdot_{0}=\bullet_{e}=45^{0}$ is satisfied, according to 1 , the power of the $\mathrm{HE}_{11}^{\mathrm{e}}$ wave is equal to the power of the $\mathrm{HE}_{11}^{\mathrm{e}}$ wave. 
Table 1: Parameters of optical fibers that deposited in a spiral line for equal separation of signal power

\begin{tabular}{|c|c|c|c|c|c|}
\hline Optical fiber content & $\begin{array}{r}\text { No. of turns of } \\
\text { the helix }(\mathrm{k})\end{array}$ & $\begin{array}{l}\text { Beat length } \\
(2 \cdot, \mathrm{m})\end{array}$ & Parameter (A) & $\begin{array}{c}\text { Pitch twist } \\
(\bullet, \mathrm{m})\end{array}$ & $\begin{array}{l}\text { Radius of modal } \\
\text { field } \mathrm{HE}_{1}(\mu \mathrm{m})\end{array}$ \\
\hline $\begin{array}{l}\text { Core } 100 \% \mathrm{SiO}_{2} \\
\text { shell } 1 \% \mathrm{~F}, 99 \% \mathrm{SiO}_{2}\end{array}$ & 2.834152 & 0.132692 & 0.553 & 0.034728 & 4.333 \\
\hline $\begin{array}{l}\text { Core } 2.2 \% \mathrm{GeO}_{2}, 3.3 \% \mathrm{~B}_{2} \mathrm{O}_{3}, 94.5 \% \mathrm{SiO}_{2} \text {; } \\
\text { shell } 1 \% \mathrm{~F}, 99 \% \mathrm{SiO}_{2} \\
\text { Core } 9.1 \% \mathrm{P}_{2} \mathrm{O}_{5}, 90.9 \% \mathrm{SiO}_{2}\end{array}$ & 3.375232 & 0.158025 & 0.553 & 0.034728 & 3.644 \\
\hline shell $3.5 \% \mathrm{~B}_{2} \mathrm{O}_{3}, 96.5 \% \mathrm{SiO}_{2}$ & 4.810408 & 0.225218 & 0.553 & 0.034728 & 2.579 \\
\hline $\begin{array}{l}\text { shell } 1 \% \mathrm{~F}, 99 \% \mathrm{SiO}_{2} \\
\text { Core } 9.1 \% \mathrm{GeO}_{2}, 7.7 \% \mathrm{~B}_{2} \mathrm{O}_{3}, 83.2 \% \mathrm{Si} \mathrm{O}_{2}\end{array}$ & 7.591813 & 0.355441 & 0.553 & 0.034728 & 1.689 \\
\hline $\begin{array}{l}\text { shell } 2.2 \% \mathrm{GeO}_{2}, 3.3 \% \mathrm{~B}_{2} \mathrm{O}_{3}, 94.5 \% \mathrm{SiO}_{2} \\
\text { Core } 4.03 \% \mathrm{GeO}_{2}, 9.7 \% \mathrm{~B}_{2} \mathrm{O}_{3}, 86.27 \% \mathrm{SiO}_{2}\end{array}$ & 3.782487 & 0.177092 & 0.553 & 0.034728 & 3.272 \\
\hline $\begin{array}{l}\text { shell } 1 \% \mathrm{~F}, 99 \% \mathrm{SiO}_{2} \\
\text { Core } 0.1 \% \mathrm{GeO}_{2}, 5.4 \% \mathrm{~B}_{2} \mathrm{O}_{2}, 94.5 \% \mathrm{SiO}_{2}\end{array}$ & 3.652326 & 0.170998 & 0.553 & 0.034728 & 3.371 \\
\hline shell $1 \% \mathrm{~F}, 99 \% \mathrm{SiO}_{2}$ & 2.981806 & 0.139605 & 0.553 & 0.034728 & 4.120 \\
\hline
\end{tabular}

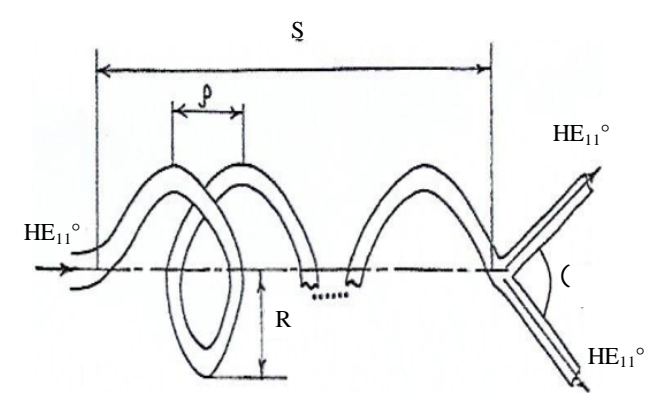

Fig. 3: Power distribution based on photo elasticity

\section{CONCLUSION}

In conclusion, we can note that the results of the studies indicate the feasibility of implementing directional couplers based on the anisotropy properties caused by photo elasticity of optical fiber, a method for calculating the parameters of the (SSMOF) for use in the fiber optic system of transfer is determined. The data obtained can also be used as a reference material for the construction of splitters based on angular dispersion, frequency-selective filters where their principle is based on the use of the properties of photo elastic optical fiber.

\section{REFERENCES}

AL-Gawagzeh, M.Y., 2009. Research the dielectric permittivity of optical fiber. J. Electron. Electr. Eng., 89: 45-49.
AL-Gawagzeh, M.Y., 2016. Studying the polarization dispersion in the bended on spiral optical waveguide. Intl. J. Commun., 1: 205-209.

Al-Gawagzeh, M.Y. and A.H.M. Rasoul, 2014. Chromatic dispersion in fiber optic with Ordered Rotating of Glass Microstructure (ORGM). Optoelectron. Adv. Mater. Rapid Commun., 8: 359-362.

Al-Gawagzeh, M.Y., M.R. Al-Hadidi and R. Al-Rzooq, 2009. Polarized modes dispersion in anisotropic optical fiber communication lines. Wseas Trans. Commun. J., 8: 11-20.

Arnold, J.M., 2001. Anisotropic effects in nonlinear optical waveguides. Wave Motion, 34: 339-352.

Iorgach, O.V., 2011. Losses on the non-uniform optical fiber cables. J. Foto Electron, 11: 61-64.

Makarov, T.V., 2004. Nonreciprocal effect waves and signals in single mode bended on spiral optical fiber. UNEERT., 1: 23-29.

Makarov, T.V., 2005. The transfer characteristic of bending optical fiber. UNEERT., 2: 29-34.

Peter, C.E., 2008. Effect of production process on the optical fiber properties. Electr. Commun. J., 2: 27-32.

Yousef, A.G.M., 2016. Possibility of minimizing the effect of transfer parameters on the transmitted signal in the spiral optical fiber. Intl. J. Commun., 1: 205-209.

Zhuck, N.P. and S.N. Shulga, 2004. Effective permittivity of a statistically inhomogeneous medium with strong permittivity fluctuations. Prog. Electromagnet. Res., 44: 169-195. 